\title{
ARTICLE
}

\section{Zinc oxide nano-enabled microfluidic reactor for water purification and its applicability to volatile organic compounds}

\author{
Imadeddine Azzouz ${ }^{1, *}$, Yamina Ghozlane Habba ${ }^{2 * *}$, Martine Capochichi-Gnambodoe ${ }^{2}$, Frédéric Marty ${ }^{1}$, Jérôme Vial ${ }^{3}$,
} Yamin Leprince-Wang ${ }^{2}$ and Tarik Bourouina ${ }^{1}$

This paper reports fast and efficient chemical decontamination of water within a tree-branched centimeter-scale microfluidic reactor. The microreactor integrates Zinc oxide nanowires (ZnO NWs) in situ grown acting as an efficient photocatalytic nanomaterial layer. Direct growth of $\mathrm{ZnO}$ NWs within the microfluidic chamber brings this photocatalytic medium at the very close vicinity of the water flow path, hence minimizing the required interaction time to produce efficient purification performance. We demonstrate a degradation efficiency of $95 \%$ in $<5 \mathrm{~s}$ of residence time in one-pass only. According to our estimates, it becomes attainable using microfluidic reactors to produce decontamination of merely 1 I of water per day, typical of the human daily drinking water needs. To conduct our experiments, we have chosen a laboratory-scale case study as a seed for addressing the health concern of water contamination by volatile organic compounds (VOCs), which remain difficult to remove using alternative decontamination techniques, especially those involving water evaporation. The contaminated water sample contains mixture of five pollutants: Benzene; Toluene; Ethylbenzene; $\mathrm{m}-\mathrm{p}$ Xylenes; and o-Xylene (BTEX) diluted in water at 10 p.p.m. concentration of each. Degradation was analytically monitored in a selective manner until it falls below 1 p.p.m. for each of the five pollutants, corresponding to the maximum contaminant level (MCL) established by the US Environmental Protection Agency (EPA). We also report on a preliminary study, investigating the nature of the chemical by-products after the photocatalytic VOCs degradation process.

Keywords: microfluidic reactor; water purification; zinc oxide nanowires; volatile organic compounds (VOCs); Benzene, Toluene, Ethylbenzene, $\mathrm{m}-\mathrm{p}$ Xylenes, o-Xylene (BTEX)

Microsystems \& Nanoengineering (2018) 4, 17093; doi:10.1038/micronano.2017.93; Published online: 26 February 2018

\section{INTRODUCTION}

Environmental pollution is a well-established major determining factor for public health: waterborne diseases continue to kill over two million lives per year ${ }^{1}$, which led The World Health Organization (WHO) to set the water quality at the top of its priority action list. Water is not only vital to all forms of life, as it is also an unavoidable precious resource in a wide range of industrial processes. On the other hand, an ever-increasing number of endogenic pollutants of different natures (biological, agricultural, pharmaceutical, and cosmetic) require active monitoring. However, this warning signal is contrasting a lot with the lack in lowcost instrumentation, which might allow efficient tools for monitoring of water quality and their accessibility at a global scale. This holds true for the measurement systems that are required for screening and quantifying the numerous water pollutants.

Besides the needs of low-cost instrumentation for measuring the amount of pollutants, the problem of chemical pollution can also be addressed through a remediation strategy of depollution and recycling; indeed water can be decontaminated in an efficient manner, involving the use of different technologies including adsorption ${ }^{2}$, microfiltration ${ }^{3}$, photo-Fenton ${ }^{4}$, and photocatalysis ${ }^{5}$. In this area, Lab-On-Chip technologies may play an important role, not only due to low cost but also due to intrinsic favorable scaling laws of physics and chemistry; ultracompact optofluidic microreactors inherit the merits of microfluidics, such as large surface/ volume ratio, easy flow control, and rapid fabrication while keeping encouraging overlook for large volume photocatalytic water treatment.

Important research efforts have been already carried out on photocatalytic water purification within microfluidic reactors ${ }^{6-10}$, also summarized in a recent review ${ }^{11}$. Still there is a margin of progress in this field in which we aim to contribute herein.

First, most previous reports relate to the use of thin films as the photocatalytic material. Only a few reports relate to nanomaterials ${ }^{12-14}$ and most often than not, the photocatalytic material is $\mathrm{TiO}_{2}{ }^{15-18}$. $\mathrm{ZnO}$ is an interesting alternative to $\mathrm{TiO}_{2}$, many research groups all over the world focused on this promising research field: developments of $\mathrm{ZnO}$-based photocatalyst in water treatment technology ${ }^{19-26}$. In a recent report of our group, $\mathrm{ZnO} \mathrm{NWs}$ were proven to be efficient photocatalyst ${ }^{27}$. However, their use was demonstrated in static mode where time constants are in the range of hours, due to the diffusion length, which affects the length-scale limit of mass transfer during the degradation process of polluted water ${ }^{28,29}$. Fortunately, it is expected that microfluidic systems can provide ultrahigh purity water by taking advantages of the appropriate size for reactiondiffusion process, possibly leading to enhance the photocatalysis

\footnotetext{
${ }^{1}$ Université Paris-Est, ESIEE-Paris, ESYCOM EA 2552, 2 Boulevard Blaise Pascal, 93162 Noisy-le-Grand, France; ${ }^{2}$ Université Paris-Est, UPEM, ESYCOM EA 2552, 5 Boulevard Descartes, 77420 Champs sur Marne, France and ${ }^{3}$ LSABM, CNRS UMR 8231, ESPCI ParisTech-PSL Research University, 10 Rue Vauquelin, 75005 Paris, France

Correspondence: Yamin Leprince-Wang (yamin.leprince@u-pem.fr)

*These authors contributed equally to this work.

Received: 21 March 2017; revised: 3 September 2017; accepted: 30 September 2017
} 
efficiency ${ }^{30-32}$. By matching the flow rate and reaction-diffusion process of the $\mathrm{ZnO}$ nanostructure under UV illumination, a practical microfluidic system based on parallel micro-nanostructured pipelines will be developed for precisely control ultrapurified water treatment.

In this report, we demonstrate the $\mathrm{ZnO} N W$ s integration within a microfluidic reactor leading to a drastic reduction of the reaction time, which dropped to a few seconds, thanks to the enhanced diffusion time, with further capabilities of fine tuning the water flow rate for proper control of the degradation efficiency. The latter is also inherently enhanced due to the ability of the water sample to flow easily through the confined reactor exhibiting large surface to volume, facilitating high throughput and highspeed interaction of water with the photocatalyst.

On the other hand, numerous demonstration of water purification reported in the literature are based on the use of model chemicals such as methylene blue (MB) and methyl orange (MO), and/or on pollutants at rather high-concentration levels. In this report, we demonstrate efficient degradation of a mixture of VOCs at the parts per million (p.p.m.) concentration levels, chosen to be initially higher than the maximum concentration limit (MCL), which allow us to address a regulated heath concern for the demonstration of our device capabilities. Several reports already highlighted the increasing occurrence of VOCs in aquifersascribed to both natural and anthropogenic factors, and the related implications on human health through drinking water. Consequently, there is an interest in quantifying those VOCs, preferably in a selective manner, though on-site sampling and analysis, for further decontamination actions when necessary. In this frame, there are several implications of our work on VOC decontamination by on-chip photocatalysis enhanced by $\mathrm{ZnO}$ NWs. First, because VOCs have a lower boiling temperature than water, they remain difficult to remove using alternative purification technologies, which involve evaporation of water and distillation. This holds true for the emerging solar-driven low-cost technologies for seawater desalination and waste-water purification. On the contrary, photocatalysis was proven to be efficient. Nowadays, removal of VOCs is currently done using carbonactivated filters. However, the efficiency of such filters requires regular maintenance. With this respect, as photocatalysis lead to decomposition of the VOC's molecules, it appears as a more sustainable technology. When implemented in the chip format, as considered in our work, it has additional potential of low cost.

\section{MATERIALS AND METHODS}

Photocatalysis studies were carried out on a nano-enabled microfluidic reactor (schematically depicted in Figure 1 and described physically in Figure 2). This reactor was placed under a ultravolet (UV) lamp of $365 \mathrm{~nm}$ (HAMAMATSU-LC8, Power $4500 \mathrm{~mW} \mathrm{~cm}^{-2}$ ) shining the sample with normal incidence. The distance UV lamp and sample has been maintained at $10 \mathrm{~cm}$ for all photocatalysis experiments. Precise control of the flow rate of water flowing through the microreaction chamber is achieved using an electrically monitored syringe pump (PHD ULTRA, Harvard apparatus) at flow rate levels ranging from 50 to $1000 \mu \mathrm{L} \mathrm{min}^{-1}$. The water samples before and after being decontaminated were analyzed using both gas chromatography (GC) and optical spectroscopy. A UV-Vis spectrometer (Lambda 35, Perkin Elmer), FT-IR spectrometer (Spectrum two, Perkin Elmer, Villebon sur Yvette, France) and gas chromatograph (Clarus 680, Perkin Elmer) have been used.

The VOC mixture of Benzene, Toluene, Ethylbenzene, m-p-o Xylenes (BTEX) was purchased from Restek (Lisses, France), containing compounds at a concentration of $2000 \mu \mathrm{g} \mathrm{mL} \mathrm{L}^{-1}$ in methanol. In order to prepare the contaminated water samples,

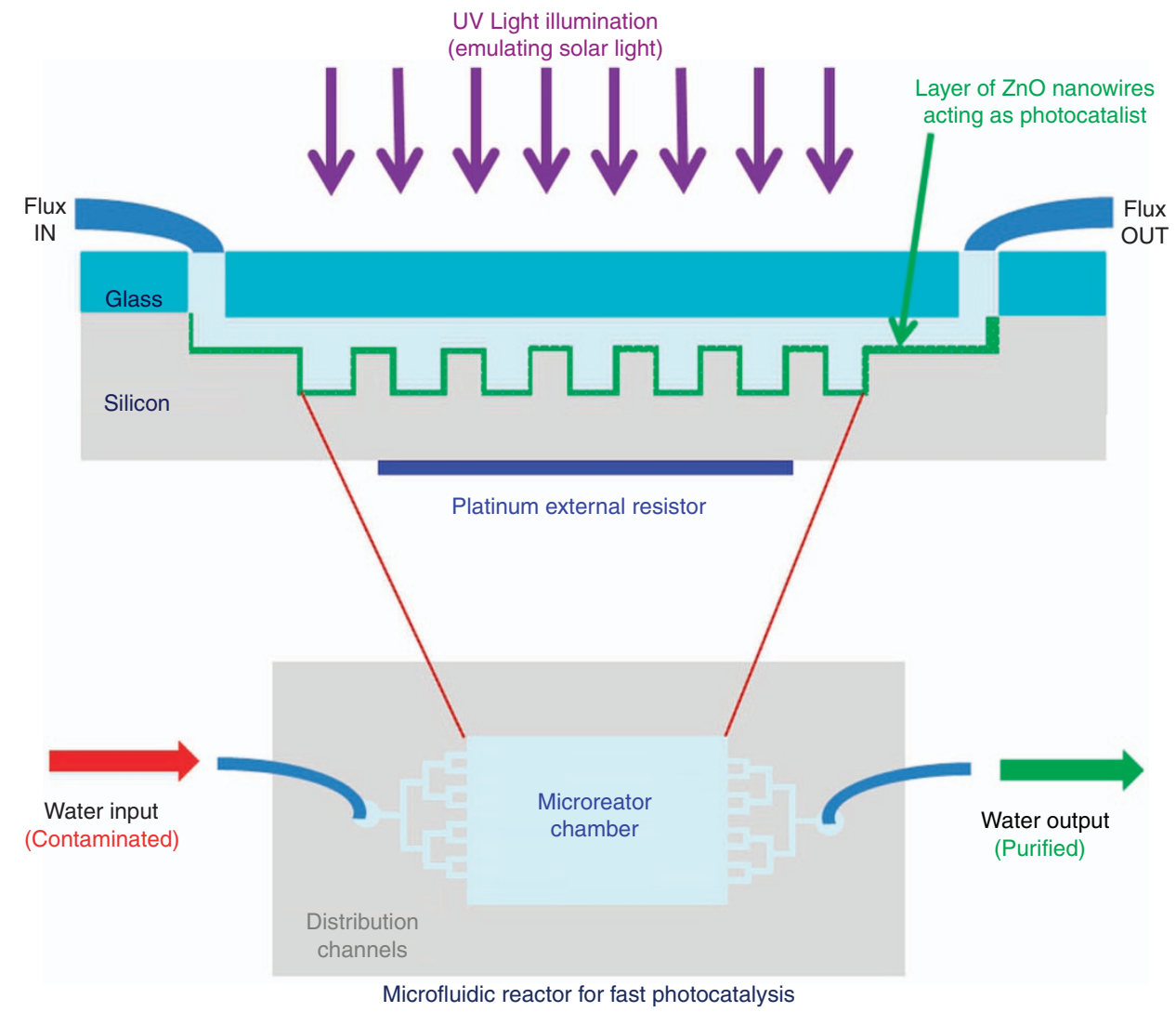

Figure 1 Schematic illustration of the experimental setup used for water decontamination. The microfluidic reaction chamber including ZnO nanowires acting as photocatalyst is exposed to ultraviolet (UV) light while water is flowing, leading to purified water at the outlet. 
a

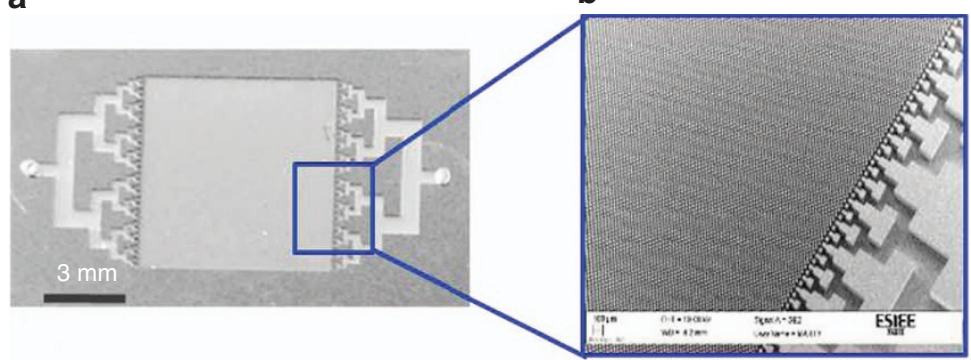

C

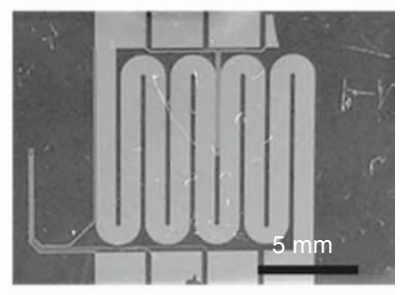

Figure 2 Micrographs showing overall views of the microreactor. (a) Top view of the reactor, (b) zoom showing the tree branches and the pillar array within the chamber, (c) backside view of the reactor incorporating platinum resistors for optional temperature control.

a

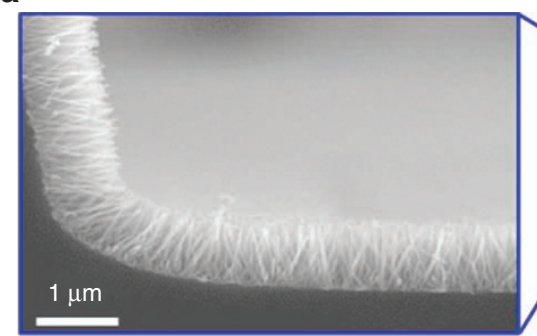

b

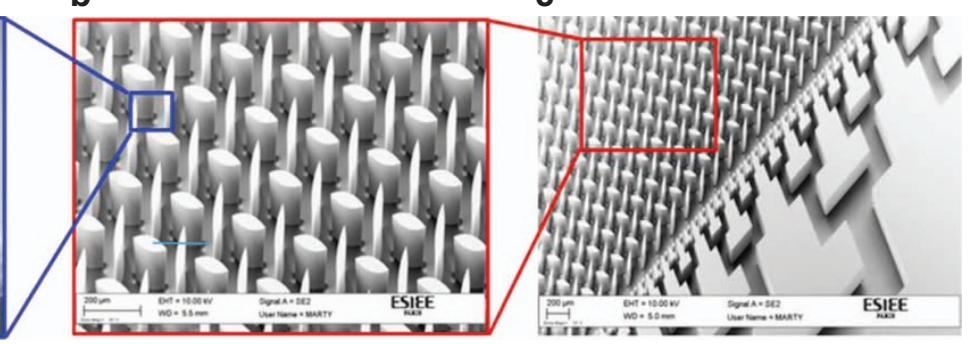

Figure 3 Hierarchical micro-nano structuration combining microscale structures obtained by deep reactive ion etching of silicon and further growth of ZnO NWs using the static method. (a) Resulting micro-nano-structure with ZnO NWs, (b) close view of the silicon microstructure, (c) view of the reactor tree-branched inlets to the chamber. ZnO NWs, Zinc oxide nanowires.

this mixture of VOCs was diluted in ultrapure water $(18 \mathrm{M} \Omega \mathrm{cm}$ resistivity) obtained from a Millipore system (Milli-Q Gradient).

A bench-scale GC was used to evaluate the efficiency of the degradation. The chromatograph was equipped with split-splitless injector set at $120^{\circ} \mathrm{C}$ and a flame ionization detector (FID) set at $250^{\circ}$ C. Nitrogen was used as a carrier gas. Hydrogen, air and nitrogen were produced from a set of gas generators (Peak scientific). Hydrogen and air gas flow rates were set at 45 and $450 \mathrm{~mL} \mathrm{~min}^{-1}$, respectively. Chromatographic separations to analyze BTEX solutions before and after degradation were achieved on fast GC column $(10 \mathrm{~m} \times 10 \mu \mathrm{m} \times 0.1 \mu \mathrm{m})$ from J\&W Scientific CP-Sil 5. A Turbomatrix 40 head space autosampler was used to introduce samples automatically directly into the GC. Chemstation software was used for the data evaluation. The morphology of $\mathrm{ZnO} N W s$ was characterized using a scanning electron microscope (SEM, NEON 40 ZEISS) operating at $10 \mathrm{kV}$ accelerating voltage.

\section{RESULTS AND DISCUSSION}

\section{Microreactor fabrication}

The microfluidic chamber of the microreactor (Figure 2a) has been fabricated on a double-sided polished silicon wafer (16 devices per 4-inch diameter silicon wafer). A microfluidic chamber including structuration with pillar arrays was fabricated by deep reactive ion etching using an anisotropic standard Bosch process leading to an etched microchamber depth of $150 \mu \mathrm{m}$. The resulting silicon microstructuration is shown in the SEM micrograph of Figures $2 \mathrm{~b}$ and 3 . In order to enhance water interaction with the walls through mixing and also to increase the total surface area of the walls, the reactor chamber is decorated with a double two-dimensional array of $120 \mu \mathrm{m}$-long almond-shaped pillars with a periodicity of $240 \mu \mathrm{m}$. Furthermore, for better homogeneity of the fluid flow inside the chamber, its input and output are designed along a tree-branched, seven-level fractal channel structure also shown in Figure 3, whose widths are 663, $389,231,134,80,45$, and $27 \mu \mathrm{m}$, respectively. The structured silicon wafer was then anodically bonded to a Pyrex substrate in order to close the chamber. Inlet and outlet access holes to the microchamber were etched on the Pyrex side. After assembly, the wafer was diced to obtain $2 \mathrm{~cm} \times 1.5 \mathrm{~cm}$ microreactor chips. To enable optional temperature programming of the microchip, two platinum filaments (one $50 \Omega$ filament for resistive heating and one $1000 \Omega$ filament for temperature sensing) were deposited by sputtering on the backside of the wafer (Figure 2c). Those optional on-board resistive heaters and temperature sensors can provide further enhancement on the control of the chemical reactions, but were not yet used in this preliminary work, where all experiments have been conducted at room temperature of $20^{\circ} \mathrm{C}$.

The silicon microstructuration shown in Figures $3 \mathrm{~b}$ and $\mathrm{c}$ aims providing an additional hierarchy level for improving the ultimate total expanded surface of the $\mathrm{ZnO} \mathrm{NWs}$ exposed to water. Figure 3a illustrates such hierarchical micro-nano structuration, which was obtained in this case by the so-called static method. In the present work, ZnO NWs were obtained by the so-called dynamic method, involving flow of the growth solution inside the microreactor described hereafter, that is after silicon was bonded to glass. In the latter case, the aqueous solutions used for NWs growth passes only through the microfluidic chamber leading to in situ and localized growth of $\mathrm{ZnO} N W s$ within the chamber.

The ZnO NWs samples have been prepared using two-step hydrothermal method similar to our previous work ${ }^{33}$, but adapted here under dynamic flow regime. The first step consists in injection of $1 \mathrm{~mL}$ buffer solution to the microchamber. For the buffer solution preparation, the zinc acetate dihydrate ( $\mathrm{ZnA}$ $\mathrm{C}_{2} \cdot 2 \mathrm{H}_{2} \mathrm{O}, 0.01 \mathrm{M}$ ) was dissolved in the absolute ethanol (99.9\%). The coated microchamber was annealed in an oven for $15 \mathrm{~min}$ at $350{ }^{\circ} \mathrm{C}$. The second step consists in also under dynamic flow regime, the $\mathrm{ZnO}$ nanorods growth via hydrothermal process at $95^{\circ} \mathrm{C}$. For the $\mathrm{ZnO}$ nanorod growth, the hydrothermal solution was prepared by mixing equimolar aqueous solutions $(0.025 \mathrm{M})$ of the zinc nitrate $\left(\mathrm{Zn}\left(\mathrm{NO}_{3}\right)_{2}\right)$ and the hexamethylenetetramine (HMTA). Control of all compositions was made by gravimetry on 
an analytical balance Mettler Toledo AM50 (accuracy $0.0001 \mathrm{~g}$ ). Figure 3a shows a typical morphology of the inner surface of microchamber decorated by the $\mathrm{ZnO}$ nanorods, which is showing an excellent crystallinity. In our previous work ${ }^{27,33}$, the microstructural properties of $\mathrm{ZnO} \mathrm{NWs}$ have been investigated by XRD, HRTEM and UV-Visible (UV-Vis) absorption spectroscopy. Typical characterization results are given in Supplementary Material S2. For convenience, those experiments have been carried out on the ZnO NWs grown on bare silicon surfaces.

\section{Water purification experiments}

After functionalization with $\mathrm{ZnO} \mathrm{NWs}$, the microfluidic chamber becomes a photocatalytic reaction chamber. The corresponding chip is then mounted to a manifold and connected to the rest of the setup via $10 \mathrm{~cm}$-long, $200 \mu \mathrm{m}$-diameter tubes.

A calibrated solution of 10 p.p.m. was prepared by suitable dilution in water of the BTEX mixture purchased from Restek. This solution was introduced in a $10 \mathrm{~mL}$ syringe and mounted on a syringe pump connected to the microreactor. The degraded BTEX water solutions were collected from the outlet and analyzed by both spectroscopic and chromatographic methods.

We started our study using UV absorption spectroscopy. We performed a comparative analysis of the contents of water sample before and after completion of the photocatalytic degradation after a single pass through the microchamber decorated with $\mathrm{ZnO}$ NWs. A control experiment was also performed using a similar microchamber, which has no $\mathrm{ZnO} N W s$. Figure $4 a$ shows the results of this comparison and Figure $4 \mathrm{~b}$ gives the UV absorption spectra of each VOC in the same wavelength range. We note that the absorption spectrum after the control experiment (dashed line in Figure 4a) is quite similar to the spectrum of the initially polluted water sample. On the contrary, the absorption decreases significantly when using the reactor that contains $\mathrm{ZnO}$ NWs. The difference before and after the photocatalysis assisted by $\mathrm{ZnO} N W s$ is clearer in the spectral range between 200 and $225 \mathrm{~nm}$, where all VOCs have the maximum absorption as shown in Figure $4 \mathrm{~b}$, even though we can also notice some decrease in the spectral range around $260 \mathrm{~nm}$. A control experiment has been performed using a similar microfluidic microchamber, which has no $\mathrm{ZnO}$ NWs. The corresponding result of the absorption spectrum recorded after UV irradiation is shown in the dashed line of Figure 4a. As it can be seen, there is no difference between this absorption spectrum with the one obtained initially on the polluted water sample, before UV irradiation. Obviously, one can conclude from this first experiment that $\mathrm{ZnO}$ NWs are responsible of the degradation process.

However, even though we have an overall reduction of the absorption magnitude, one cannot distinguish in a selective manner by which VOCs have been mostly affected by the photocatalysis. In order to provide an answer, we made additional analysis using chromatography as described hereafter. One can also notice from Figure $4 a$ that besides the decrease of absorption after the purification step, we also have additional peaks that appear. Indeed, the absorption spectrum extends above $225 \mathrm{~nm}$ until $235 \mathrm{~nm}$, suggesting that chemical by-products may have been formed during the photocatalytic reaction. Nevertheless, those by-products cannot be identified at this stage as UV absorption spectroscopy appears not selective enough to discriminate all compounds.

For the chromatography analysis of the BTEX compounds dissolved in water, a method based on the use of GC analysis was developed using static head space. The conditions were optimized with respect to sample thermostating time and temperature. Figure 5a shows superimposition of two chromatograms before and after one-pass degradation under optimal conditions $\left(50 \mu \mathrm{L} \mathrm{min}^{-1}\right.$ flow rate). This enables in a selective manner that all VOCs have been dropped below the threshold concentration level of 1 p.p.m., which is equivalent to the MCL established by US $\mathrm{EPA}^{34}$. Figure $5 \mathrm{~b}$ shows repeatability measurement after a onepass photocatalysis showing almost the same contents after three experiments. Furthermore, Supplementary Material S1 is provided in order to further support the fact that $\mathrm{ZnO} N W s$ has an important photocatalytic activity, which can be extended not only to the five VOCs considered in this work, but also to three other commonly used chemical dyes, namely methylene blue (MB), methyl orange (MO), and Acid Red 14 (AR14). These results are also supported by control experiments. The presented results on Acid Red 14 (AR14) also show that ZnO NWs has an important photocatalytic activity even at much higher concentrations than the p.p.m. level, as considered in this work. Indeed, the results shown in Supplementary Material S1 are related to concentration levels of $10 \mathrm{mM}$ (> 5000 p.p.m. for AR14). Furthermore, the results shown in Supplementary Material S1 illustrate the fact that photocatalytic activity of $\mathrm{ZnO} N W s$ is proven to be repeatable over time, as the degradation efficiency is shown to be constant after several repeated cycles of $3 \mathrm{~h}$ each. $\mathrm{ZnO}$ NWs are eventually found to be stable over time in aqueous environment. It is worth mentioning that potential dissolution and photodissolution of $\mathrm{ZnO}$ has been reported in the literature ${ }^{35}$. It was found that these effects occur at low pH values, below 5. In all our experiments, we made sure that water was neutral, with $\mathrm{pH}$ values ranging from 6 to 7.

One can legitimately pose the question about the practical usefulness of such microfluidic decontamination devices. A simple calculation of orders of magnitudes can highlight the fact that if one uses a flow rate of $700 \mu \mathrm{L} \mathrm{min}{ }^{-1}$ instead of $50 \mu \mathrm{L} \mathrm{min}{ }^{-1}$, it is attainable to achieve decontamination of 11 of water in only
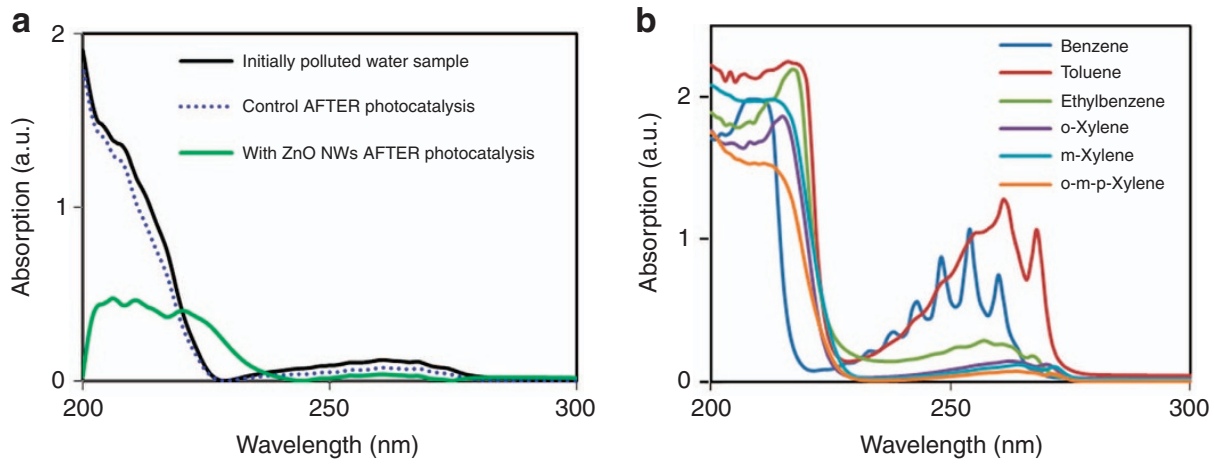

Figure 4 (a) Comparative analysis by ultraviolet (UV) absorption spectroscopy of a water sample before and after a single-pass photocatalysis within the microreactor containing Zinc oxide nanowires ( $\mathrm{ZnO} N W s$ ). The results of a control experiment (without ZnO NWs) are shown in dashed line. (b) Reference absorption spectra of volatile organic compounds (VOCs) of interest. 

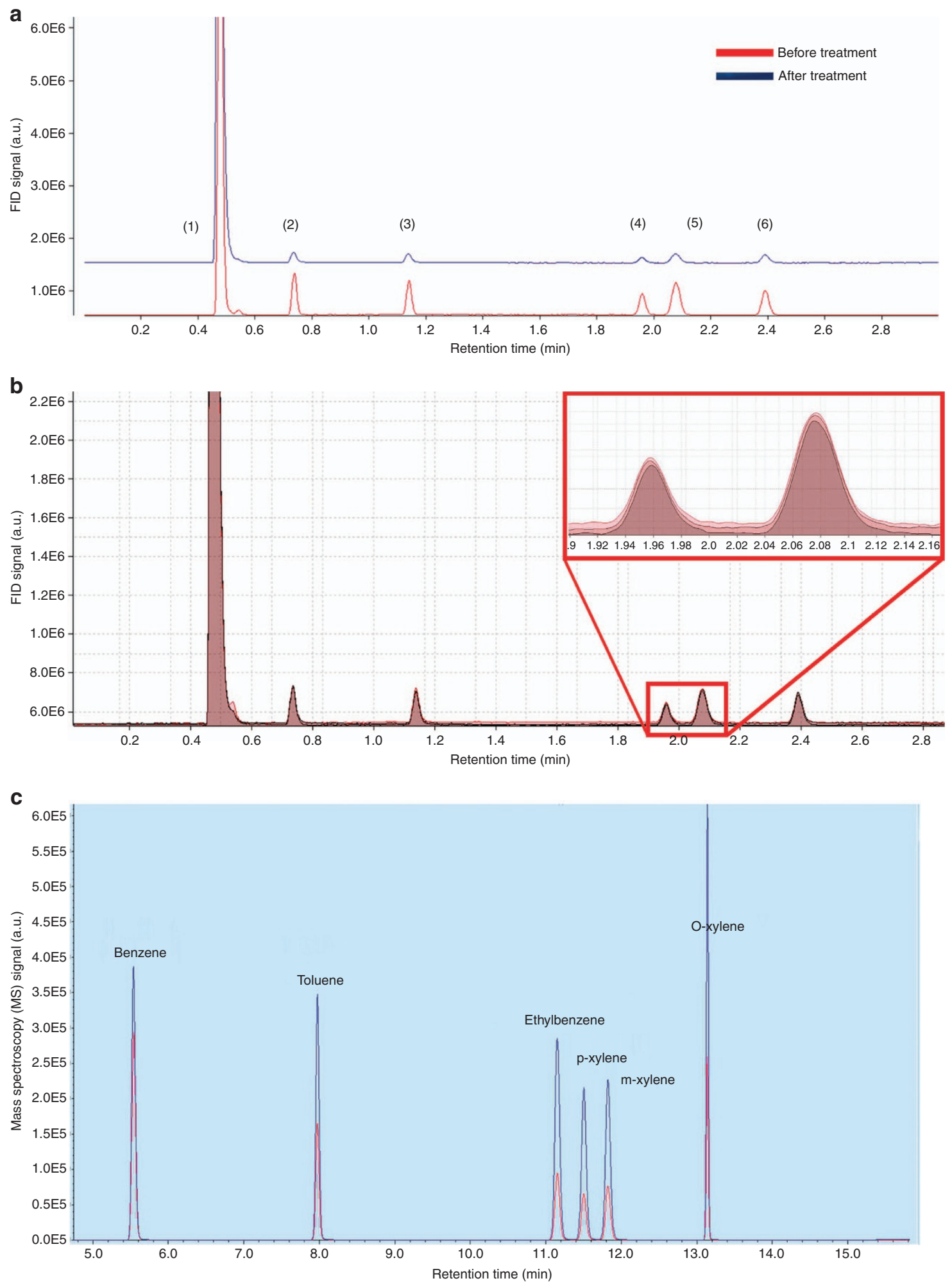

Figure 5 Analysis by gas chromatography (GC). (a) Comparative measurement obtained by GC-FID of the water contents before and after a one-pass photocatalysis within the microreactor with a flow rate of $50 \mu \mathrm{L} \mathrm{min}^{-1}$, a nearly $10 \times$ reduction of the concentration can be noticed after purification for each of the five volatile organic compounds (VOCs). (b) Repeatability measurement after a one-pass photocatalysis showing almost the same contents after three experiments. (c) Comparative measurement obtained by GC-MS of the water contents before and after a one-pass photocatalysis within the microreactor with a flow rate of $250 \mu \mathrm{L} \mathrm{min}{ }^{-1}$ of the head-space fraction, showing no evidence of by-products in the explored range. Initial content of water is a mixture of BTEX at a concentration level of 10 p.p.m. for each of the five VOC compounds initially diluted in methanol solvent (1), benzene-(2), toluene-(3), ethylbenzene-(4), m-p xylene-(5) and o-xylene-(6). 
1 day, which is consistent with the order of magnitude of daily drinking water needs of humans. The only parameter that needs to be adjusted with respect to the previous experiment is the flow rate, hence the importance of studying the effect of this parameter. A trade-off will certainly need to be found between flow rate and the acceptable degradation efficiency because the flow rate is one of the major factors that affects the degradation efficiency in the microreactor system. The flow rate is indeed related to the effective residence time (that is, the degradation time) of the contaminated solution in the reaction chamber by the following relationship:

$$
\text { Effective residence time }=\frac{\text { Chamber volume }}{\text { Flow rate }}
$$

To investigate the effect of the flow rate, the solutions are pumped at the different flow rates of $50,100,250,500$, and $1000 \mu \mathrm{L} \mathrm{min}^{-1}$, respectively. The corresponding values for the effective residence time (that is, the degradation time) are $8.8,4.4,1.8,0.9$, and $0.4 \mathrm{~s}$, respectively. The residual percentage of non-degraded pollutants is plotted in Figure 6 as a function of flow rate. The data histograms and the error bars represent the averaged values and the s.d. following three repeated measurements, respectively.

The degradation percentage increases with the effective residence time and reaches an average of 95\% (corresponding to the flow rate $50 \mu \mathrm{L} \mathrm{min}{ }^{-1}$ ). The trend of these results was expected because the longer the residence time is, the more photoreaction occurs. It is also not surprising that the degradation is not linear with respect to residence time as a saturation trend is expected at the ultimate an asymptotic value of $100 \%$. Figure 6 also depicts the differences in term of degradation efficiency between the five considered VOCs at a given flow rate. For example, at a flow rate of $1000 \mu \mathrm{L} \mathrm{min}{ }^{-1}, 51.3 \%$ of benzene is degraded compared to $60.7 \%$ of degraded ethylbenzene.

Dead volume and related retention time are important performance metrics in continuous mode purification. In our case, the dead volume is the microchamber volume itself. Indeed, nearly all the volume of the reactor can be occupied by water because the height of the $\mathrm{ZnO}$ NWs does not exceed $1 \mu \mathrm{m}$, while the height of the microreactor chamber is $150 \mu \mathrm{m}$ and its lateral dimensions are $7 \times 7 \mathrm{~mm}$, corresponding to a volume of $7.4 \mu \mathrm{L}$. When combining with the flow-rate values considered in our work, this leads to a retention time ranging from $441 \mathrm{~ms}$ to $8.8 \mathrm{~s}$, respectively, for flow rates of 1000 and $50 \mu \mathrm{L} \mathrm{min}^{-1}$. According to the best purification performance obtained at a flow rate of $50 \mu \mathrm{L} \mathrm{min}{ }^{-1}$, one can extrapolate the fact that a reaction time of at least $8.8 \mathrm{~s}$ is sufficient for the case study under consideration in this paper, which is also consistent with the well-known advantages of microfluidic reactors in terms of reduced reaction time. It is worth mentioning that these estimates of the retention time are consistent with the volumes of purified water in a 5-s single-pass, which ranges from 5 to $80 \mu \mathrm{L}$ depending on the flow rate.

According to our rather recent experience in water decontamination using $\mathrm{ZnO} \mathrm{NWs}$, we found that reaching degradation percentages in the order of $90-95 \%$ require different times depending on the reactor size. This time ranges from hours, minutes, and then seconds, for reactors whose characteristic lengths are in the order of $10 \mathrm{~cm}, 1 \mathrm{~mm}$, and $100 \mu \mathrm{m}$, respectively. This scaling trend suggests that it is a diffusion-limited process, which governs photocatalysis with $\mathrm{ZnO}$ NWs.

After the above quantification of the device performance in terms of degradation efficiency and transit time, we started pushing our investigation further. In addition to the residual amounts of the five pollutants of the initial water sample, we were indeed expecting to see other by-products of the photocatalysis. But obviously, one can see from the upper chromatogram shown in Figure 5a that only those residual five pollutants can be found, at least using Micro-GC coupled with a flame ionization detector (GC-FID). We then made new analysis using GC, but in this case, it was combined with mass spectrometry, which provides better selectivity and better signal to noise ratio. The corresponding chromatograms are superimposed in Figure $5 \mathrm{c}$. They relate to a water sample analyzed before and after photocatalytic degradation at a flow rate of $250 \mu \mathrm{L} \mathrm{min}{ }^{-1}$. Here again, one can clearly observe from these chromatograms that only the five VOCs can be seen and there is no evidence of by-products that may have been formed during the photocatalysis, at least in the explored range. Similar results were obtained at different flow rates. It is therefore tempting to conclude at this stage that the photocatalysis of the five VOC pollutants led to a complete degradation of the VOC molecules. However, further experiments and investigations are still needed and will be conducted in the near future to elaborate more on the nature of the by-products formed during the photocatalytic reaction.

\section{CONCLUSION}

In this work, a nano-enabled photocatalytic microreactor for water purification was successfully fabricated by in situ growth of $\mathrm{ZnO}$ nanowires within a microfluidic chamber $(7 \mathrm{~mm} \times 7 \mathrm{~mm} \times 150 \mu \mathrm{m})$. According to the large surface to volume ratio of the $\mathrm{ZnO}$ nanowires and their proximity with the water flow within the microfluidic chamber, the proposed microreactor was proven to be very efficient and fast, as
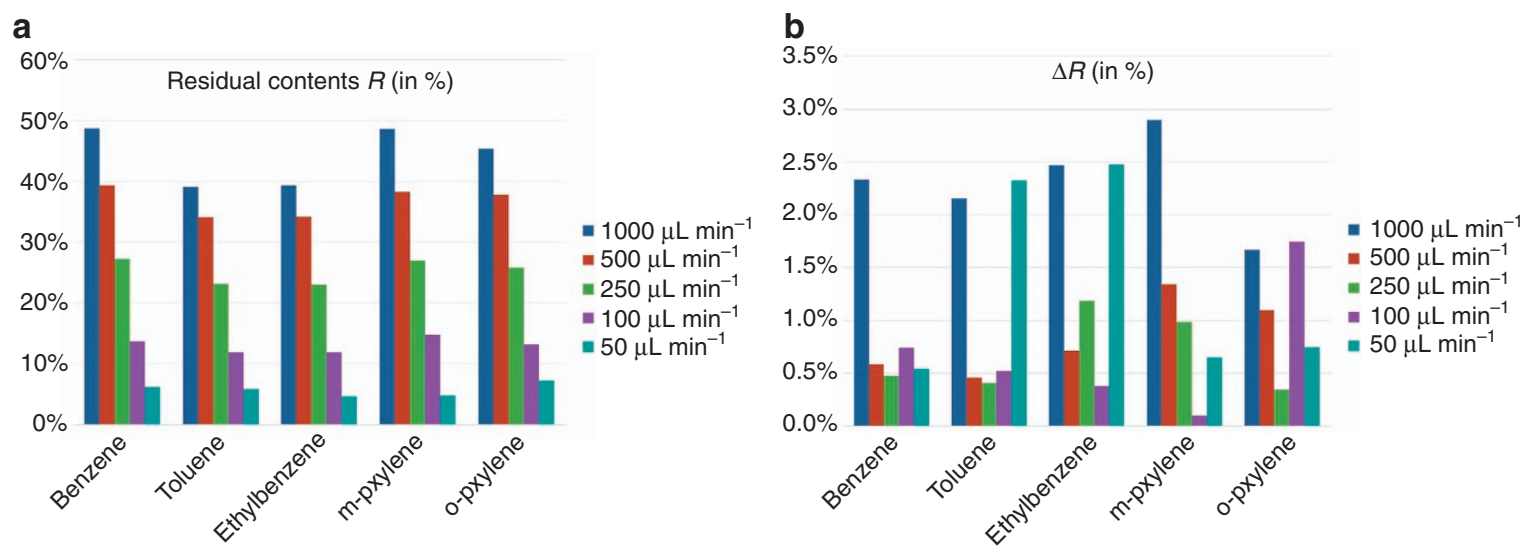

Figure 6 (a) Residual contents $R$ (in \%) and (b) corresponding errors $\Delta R$ (in \%), for each VOC of the BTEX mixture after one-pass photocatalytic purification for different values of the flow rate (from 50 to $1000 \mu \mathrm{L} \mathrm{min}{ }^{-1}$ ) calculated from peak areas (obtained by GC-FID). All measures were repeated three times, errors bars correspond to the s.d. 
degradation up to $95 \%$ was achieved in $<5 \mathrm{~s}$. When considering a flow rate of $250 \mu \mathrm{L} \mathrm{min}^{-1}$, a realistic throughput is evaluated enabling the purification of merely $0.4 \mathrm{I}$ per day. This estimate is based not only on the measured degradation efficiency versus flow rate, but it is also supported by the fact that $\mathrm{ZnO} N W s$ photocatalytic activity remains stable over time (cf. Supplementary Material S1). The purification process of water contaminated with a BTEX mixture was monitored and characterized through both chromatographic and optical spectroscopy measurements. The subsequent research work will focus first on determination of the chemical structure of the by-product(s), revealed by our preliminary results. Moreover, stability and recyclability being crucial performance metrics of the catalyst, our detailed study conducted on $\mathrm{ZnO} N W$ s obtained by the static method will be extended to the newly proposed catalytic bed obtained through in situ grown $\mathrm{ZnO} \mathrm{NWs}$ within the microfluidic reactor, following methods inspired by prior works in this area ${ }^{36}$.

\section{ACKNOWLEDGEMENTS}

We thank Fanny Delille, José Dugay and Didier Thiebault from ESPCl, for their help in GC-MS measurements. This work has received funding from the ANR EquipEx SENSECITY project, the FUI 18 MIMESYS funded by Region lle-de-France and the European Union's H2020 Programme for research, technological development and demonstration under grant agreement No 644852 .

\section{COMPETING INTERESTS}

The authors declare no conflict of interest.

\section{REFERENCES}

1 World Health Organization (WHO). Sixty-fourth world health assembly: Daily notes on proceedings, 2011. Available at http://www.who.int/mediacentre/events/2011/ wha64/journal/en/index5.html.

2 Sarker M, Bhadra BN, Seo PW et al. Adsorption of benzotriazole and benzimidazole from water over a Co-based metal azolate framework MAF-5(Co). Journal of Hazardous Materials 2017; 324: 131-138.

3 Yasukawa M, Mishima S, Shibuya $M$ et al. Preparation of a forward osmosis membrane using a highly porous polyketone microfiltration membrane as a novel support. Journal of Membrane Science 2015; 487: 51-59.

4 Shestakova M, Sillanpää M. Removal of dichloromethane from ground and wastewater: A review. Chemosphere 2013; 93: 1258-1267.

5 Leblebici ME, Stefanidis GD, Van Gerven T. Comparison of photocatalytic spacetime yields of 12 reactor designs for wastewater treatment. Chemical Engineering and Processing: Process Intensification 2015; 97: 106-111.

6 Parmar J, Jang S, Soler L et al. Nano-photocatalysts in microfluidics, energy conversion and environmental applications. Lab on a Chip 2015; 15: 2352-2356.

7 Ahsan SS, Gumus A, Erickson D. Redox mediated photocatalytic water-splitting in optofluidic microreactors. Lab on a Chip 2013; 13: 409-414.

8 Wang N, Zhang X, Chen B et al. Microfluidic photoelectrocatalytic reactors for water purification with an integrated visible-light source. Lab on a Chip 2012; 12: 3983-3990.

9 Lei L, Wang N, Zhang XM et al. Optofluidic planar reactors for photocatalytic water treatment using solar energy. Biomicrofluidics 2010; 4: 043004.

10 Meng Z, Zhang X, Qin J. A high efficiency microfluidic-based photocatalytic microreactor using electrospun nanofibrous $\mathrm{TiO}_{2}$ as a photocatalyst. Nanoscale 2013; 5: 4687-4690.

11 Wang N, Zhang $X$, Wang $Y$ et al. Microfluidic reactors for photocatalytic water purification. Lab on a Chip 2014; 14: 1074-1082.

$12 \mathrm{Kim} \mathrm{J}$, Lee $\mathrm{CW}$, Choi W. Platinized $\mathrm{WO}_{3}$ as an environmental photocatalyst that generates $\mathrm{OH}$ radicals under visible light. Environmental Science \& Technology 2010; 44: 6849-6854.

13 Qu X, Alvarez PJJ, Li Q. Applications of nanotechnology in water and wastewater treatment. Water Research 2013; 47: 3931-3946.

14 Das S, Srivastava VC. Microfluidic-based photocatalytic microreactor for environmental application: A review of fabrication substrates and techniques, and operating parameters. Photochemical \& Photobiological Sciences 2016; 15: 714-730.

15 Lamberti A. Microfluidic photocatalytic device exploiting PDMS/TiO2 nanocomposite. Applied Surface Science 2015; 335: 50-54.
16 Oyama T, Aoshima A, Horikoshi S et al. Solar photocatalysis, photodegradation of a commercial detergent in aqueous $\mathrm{TiO}_{2}$ dispersions under sunlight irradiation. Solar Energy 2004; 77: 525-532.

17 Macak JM, Zlamal M, Krysa J et al. Self-organized $\mathrm{TiO}_{2}$ nanotube layers as highly efficient photocatalysts. Small 2007; 3: 300-304.

18 Tsuchiya N, Kuwabara K, Hidaka A et al. Reaction kinetics of dye decomposition processes monitored inside a photocatalytic microreactor. Physical Chemistry Chemical Physics 2012; 14: 4734-4741.

19 Hamid SBA, Tech SJ, Lai CW. Photocatalytic water oxidation on ZnO: A review. Catalysts 2017; 7: 93-106.

20 Lee KM, Lai CW, Ngai KS et al. Recent developments of zinc oxide based photocatalyst in water treatment technology: A review. Water Research 2016; 88: 428-448.

21 Srivastava VC. Photocatalytic oxidation of dye bearing wastewater by iron doped zinc oxide. Industrial \& Engineering Chemistry Research 2013; 52: 17790-17799.

22 Yan X, Zou C, Gaoc X et al. ZnO/TiO2 core-brush nanostructure: processing, microstructure and enhanced photocatalytic activity. Journal of Material Chemistry 2012; 22: 5629-5640.

23 Baruah S, Jaisai M, Imani R et al. Photocatalytic paper using zinc oxide nanorods. Science and Technology of Advanced Materials 2010; 11: 055002.

24 Amine Khoudja A, Sehili T, Pilichowski J-F et al. Photocatalytic degradation of 2 phenylphenol on $\mathrm{TiO}_{2}$ and $\mathrm{ZnO}$ in aqueous suspensions. Journal of Photochemistry and Photobiology A: Chemistry 2001; 141: 231-239.

25 Daneshvar N, Salari D, Khataee AR. Photocatalytic degradation of azo dye acid red 14 in water on $\mathrm{ZnO}$ as an alternative catalyst to TiO2. Journal of Photochemistry and Photobiology A: Chemistry 2004; 162: 317-322.

26 Saikia L, Bhuyan D, Saikia M et al. Photocatalytic performance of ZnO nanomaterials for self sensitized degradation of malachite green dye under solar light. Applied Catalysis A: General 2015; 490: 42-49.

27 Habba YG, Capochichi-Gnambodoe M, Serairi L et al. Enhanced photocatalytic activity of $\mathrm{ZnO}$ nanostructure for water purification. physica status solidi (b) 2016 ; 253: $1480-1484$.

28 Van Gerven T, Mul G, Moulijn J et al. A review of intensification of photocatalytic processes. Chemical Engineering and Processing: Process Intensification 2007; 46: 781-789.

29 Lin H, Valsaraj KT. Development of an optical fiber monolith reactor for photocatalytic wastewater Treatment. Journal of Applied Electrochemistry 2005; 35: 699-708.

30 Gorges R, Meyer S, Kreisel G. Photocatalysis in microreactors. Journal of Photochemistry and Photobiology A: Chemistry 2004; 167: 95-99.

31 Corbel S, Becheikh N, Roques-Carmes $\mathrm{T}$ et al. Mass transfer measurements and modeling in a microchannel photocatalytic reactor. Chemical Engineering Research and Design 2014; 92: 657-662.

32 Baruah A, Jindal A, Acharya $C$ et al. Microfluidic reactors for the morphology controlled synthesis and photocatalytic study of $\mathrm{ZnO}$ nanostructures. Journal of Micromechanics and Microengineering 2017; 27: 035013.

33 Chevalier-César C, Capochichi-Gnambodoe M, Leprince-Wang Y. Growth mechanism studies of $\mathrm{ZnO}$ nanowire arrays via hydrothermal method. Applied Physics A 2014; 115: 953-960.

34 Rowe BL, Toccalino PL, Moran MJ et al. Occurrence and potential human-health relevance of volatile organic compounds in drinking water from domestic wells in the United States. Environmental Health Perspectives 2007; 115: 1539-1546.

35 Han J, Qiu W, Gao W. Potential dissolution and photo-dissolution of $\mathrm{ZnO}$ thin films. Journal of Hazardous Materials 2010; 178: 115-122.

36 Sun HB, Ai YJ, Li D et al. Bismuth iron oxide nanocomposite supported on graphene oxides as the high efficient, stable and reusable catalysts for the reduction of nitroarenes under continuous flow conditions. Chemical Engineering Journal 2017; 314: 328-335.

This work is licensed under a Creative Commons Attribution 4.0 International License. The images or other third party material in this article are included in the article's Creative Commons license, unless indicated otherwise in the credit line; if the material is not included under the Creative Commons license, users will need to obtain permission from the license holder to reproduce the material. To view a copy of this license, visit http://creativecommons.org/licenses/ by/4.0/

(c) The Author(s) 2018

Supplementary Information for this article can be found on the Microsystems \& Nanoengineering website (http://www.nature.com/ micronano). 